EPiC Series in Engineering
Volume 1, 2018, Pages 50-59
$\begin{gathered}\text { ReVo 2017: Laval Virtual ReVolution } 2017 \\ \text { "Transhumanism++" }\end{gathered}$

\title{
Monster Cleaner: a serious game to learn cleaning
}

\author{
Takayuki Kosaka $^{1 *}$ and Mitsunori Matsushita ${ }^{2}$ \\ Kanagawa Institute of Technology, Japan \\ ${ }^{2}$ Knsai University,Japan. \\ kosaka@kosaka-lab.com, m_mat@kansai-u.ac.jp
}

\begin{abstract}
Children generally do not like cleaning their rooms. To change their behavior, we propose a serious game that makes cleaning fun and motivates children to clean their room. In our game "Monster Cleaner," children collect virtual monsters by sucking dust using a vacuum cleaner-like device. Two people participate in the game in a competitive manner. The more dust a player cleans, the more monsters the player will collect. The system not only makes children clean their room in a fun manner but also teaches them to the use of a vacuum cleaner. The system was demonstrated in an exhibition and the observation of game play suggested that our system can make cleaning enjoyable for children and help them learn to use a vacuum cleaner.
\end{abstract}

\section{Introduction}

Nowadays, the number of children who do not help with housework such as sweeping, washing, and dish cleaning is increasing. While many children like playing games, few children like housework. This is because they have a negative feeling about housework, such as "it is troublesome," or "it isn't fun." Housework tends to be monotonous, boring, and painful work, but it is indispensable for our daily life. We believe that it is necessary to develop technologies that will dispel the negative image of housework and let children help with housework of their own motivation. To achieve this, this study focuses on sweeping and proposes a system named the "Monster Cleaner." The system not only makes children clean their room in a fun manner but also teaches them how to use a vacuum cleaner.

\footnotetext{
${ }^{*}$ Masterminded EasyChair and created the first stable version of this document
} 


\section{Related works}

Many studies have been conducted to impart entertainment in cleaning and housework.

The interactive vacuum cleaner proposed by Ogasawara et al. [1] projects a virtual object using a projector. The system sucks the virtual object with a vacuum cleaner-like device, and such game-like interaction makes cleaning fun to the user. The "VACUUU $(\cdot \forall \cdot)$ UUUM" system developed by Tada et al. [2] extends the sucking feeling of a vacuum cleaner. This system projects a large object on the floor that cannot be sucked by a real vacuum cleaner. A vacuum cleaner-like device sucks the projected virtual dust. In accordance with the size of the virtual dust sucked, the system presents a sucking-like feeling to the user by changing the shape of the hose of the device, giving vibration feedback, and deforming the projected object.

These systems try to make cleaning enjoyable by using a special vacuum cleaner. They, however, use mechanisms for sucking virtual objects and dust, projected using a projector, and the real dust remains scattered on the floor. The room does not become clean even if a pile of virtual dust is collected. This is also a matter of teaching good habits. For instance, even if trash is left on the floor, children may ignore it and only suck virtual garbage projected by the projector. The role of a vacuum cleaner is to collect "real dust" and it is important to make the cleaning activity fun without impairing its main function.

Oono et al. have proposed a small robot that can be attached to an arbitrary appliance [3]. The robot can express the state of the appliances in motion. Their research intends to make monotonous housework pleasurable. In addition, studies on display robots [4] have been conducted to describe the function of home appliances by anthropomorphizing them by attaching pseudo eyes and arms to them. These attempts claim that imparting enjoyment and presenting information should be done without impairing the functions of conventional vacuum cleaners and home appliances.

We have been examining the use of behavior of people in the real world as game content to lead their action in the real world by stimulating their intention and interest.

For example, we have proposed a serious game for dietary education that intends to improve nutrition of children [5][6][7]. The game uses the behavior of eating foods in the real world as the conditions of the game; in this game, the player's actions such as "eating," "chewing," and "smiling" are used. To beat monsters that appear on the game screen, the player has to eat his/her least favorite food such as green peppers, tomatoes, and carrot cookies. By acting on nutritional advice during the game such as "It seems better to drink tomato juice to beat Dracula," many players tend to eat their least favorite foods. This behavior surprises the parents of the players because their children eat nutritional food without being forced. Since children are motivated to advance to higher levels in the game and beat it, they overcome their dislike toward food. As this example shows, it is possible to use serious games for training people to change their daily behavior.

With this background, this study proposes a serious game that uses cleaning in the real world as game content without losing the original purpose of the vacuum cleaner to induce children to clean their room in the real world.

\section{Design principle}

This study proposes a serious game in which children collect virtual monsters by sucking dust using a vacuum cleaner-like device. The system intends to alter children's behavior to make them enjoy cleaning. The game objective is to suck dust in the real world by using the vacuum cleaner-like device. Various events occur in the game depending on the type and place of the dust collected in the real world.

The system was designed with the following guidelines. 


\subsection{The system should clean in the real world}

In a game with "cleaning" as the theme, the vacuum cleaner should collect dust in the real world, instead of collecting virtual trash. Therefore, it is important to create games that make use of the original functions of the vacuum cleaner.

\subsection{The base cleaner should not be modified}

For ease of manufacturing, the proposed system uses a commercially available vacuum cleaner. If a special modification is performed on the vacuum cleaner, its warrant is voided. Thus, it is important not to conduct any special modification on the cleaner. In this system, all devices including the sensor and display are connected as commonly used attachments, which are made using a 3D printer. Thus, the system can be attached to any general vacuum cleaner that you may already have.

\subsection{The system should make the player learn to use the cleaner}

This system is a serious game that makes players learn to use a vacuum cleaner. In general, vacuum cleaners should be moved slowly. The method of performance measurement for home electric cleaners given by Japan Industrial Standards (JAS) regulates the moving speed of the cleaner's head to a maximum of $50 \mathrm{~cm} / \mathrm{s}$ [8]. By considering this, the system is made such that it cannot suck monsters if the speed is more than $50 \mathrm{~cm} / \mathrm{s}$. This teaches the players to keep the speed below $50 \mathrm{~cm} / \mathrm{s}$.

Also, when cleaning a Japanese tatami mat, it is important to vacuum along the grain of the mat. To teach children how to clean a tatami mat, the Monster Cleaner system can be set such that no monsters are sucked unless the vacuum is pushed along the grain of the mat. In this way, children can learn to use a vacuum cleaner properly by playing a game.

\section{Monster Cleaner}

The primary objective of the game is to clean a room by collecting dust in the real world using a vacuum cleaner. Two people participate in the game in a competitive manner. The player activates the vacuum by depressing the cleaner's trigger. Players cannot advance in the game unless they suck dust in the real world. The more dust a player sucks, the more monsters the player will collect. Points are added according to the amount of dust collected, the area cleaned, and the speed of the cleaner's head. The player with the higher score wins the game.

The monsters sucked are collected via a hosepipe in a backpack that the player carries on his/her back. By using full-color light emitting diodes (LEDs) and vibration motors, an impression is presented that the monsters are resisting being vacuumed.

Apart from ordinary monsters, the game also contains hidden boss monsters to guide the player to the intended place. The player performs cleaning while looking for a boss by checking a boss sensor mounted on the tip of Monster Cleaner. The boss sensor indicates the distance and direction to the nearest boss monster. During the configuration of the game, the boss monsters can be placed at an arbitrary position. For example, if you set the number of boss monsters in the living room higher, it is expected to make the player intensively clean the living room while searching for a boss monster.

\subsection{System configuration}

Figure 1 shows the system configuration of Monster Cleaner. Monster Cleaner comprises a vacuum device for sucking dust in the real world, a sensor device to measure the amount of dust collected, a 
display device for presenting monsters collected in proportion to the collected dust, and a control device for controlling the game.

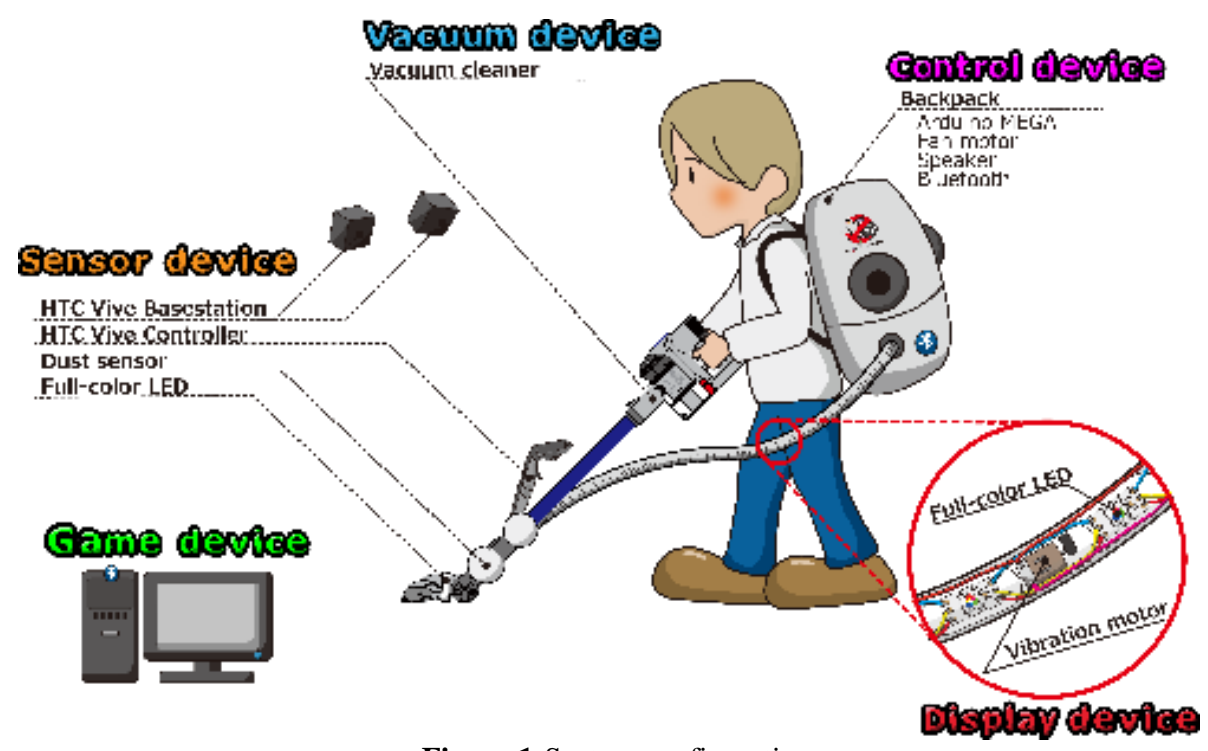

Figure 1:System configuration

\subsection{Vacuum device}

The vacuum device is a generic vacuum cleaner for collecting real dust. For not disturbing the player's sweeping behavior, we utilized the Dyson DC45 cordless vacuum cleaner. DC45 sucks dust while the trigger is pressed and stops sucking if the trigger is released. We did not conduct any special modification on the cleaner. Connection with the sensor device is achieved using an attachment made by a $3 \mathrm{D}$ printer.

\subsection{Sensor device}

A dust sensor GP2Y1010AU (Sharp Corporation) and a VIVE controller (HTC Corporation) are attached to the vacuum cleaner's head. The dust sensor measures the amount of dust sucked. It can detect dust particles larger than PM 0.8 by using a photodetection mechanism. The VIVE controller determines the angle and position of the system. It detects infrared light emitted from two base stations. These base stations are located around the floor at different positions and emit infrared light. The VIVE controller recognizes the position by receiving the light. In the normal setting, the VIVE controller can be used in a $4 \mathrm{~m} \times 3 \mathrm{~m}$ area. The dust sensor is packed into a sphere-shaped unit and attached at the neck part of the cleaner's head (see Figure 2) in the form of a common cleaner attachment. This unit also has full-color LEDs to indicate the distance to the nearest boss monster. The player can look for the boss monster by looking at the LEDs.

By combining the position-detection function of the VIVE controller with the dust sensor, the system can visualize the room and the amount of dust that has been collected.

The boss sensor, which detects the distance and direction to the boss monster from the current location, is installed in the vacuum cleaner's head. 

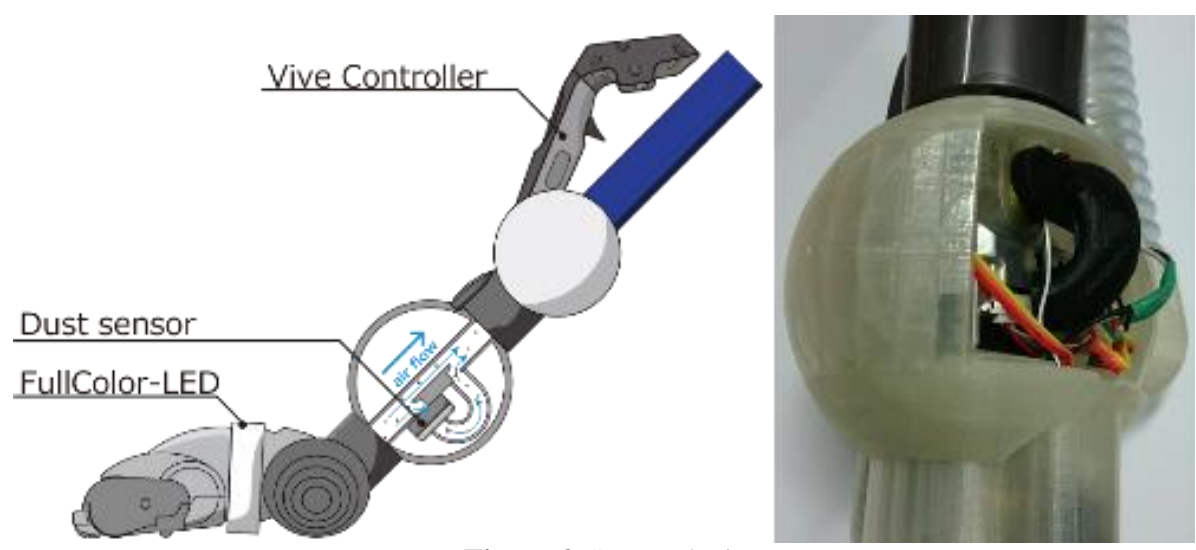

Figure 2:Sensor device

\subsection{Display device}

In Monster Cleaner, the number of monsters collected is proportional to the amount of dust collected. The sucked dust is stored in the vacuum device. At the same time, the collected monsters are stored in a backpack through a hosepipe. To visualize the monsters being sucked with the dust, the system presents a flow of light along the hosepipe. To do this, forty full-color LEDs and eight vibration motors are installed in the hosepipe (see Figure 3). The system presents the shape of sucking monsters with the flow light, vibrations, and sounds. The LEDs turn blue if not much dust is sucked and red if the sucked dust is substantial. Players can intuitively understand the amount of dust sucked by looking at the color.

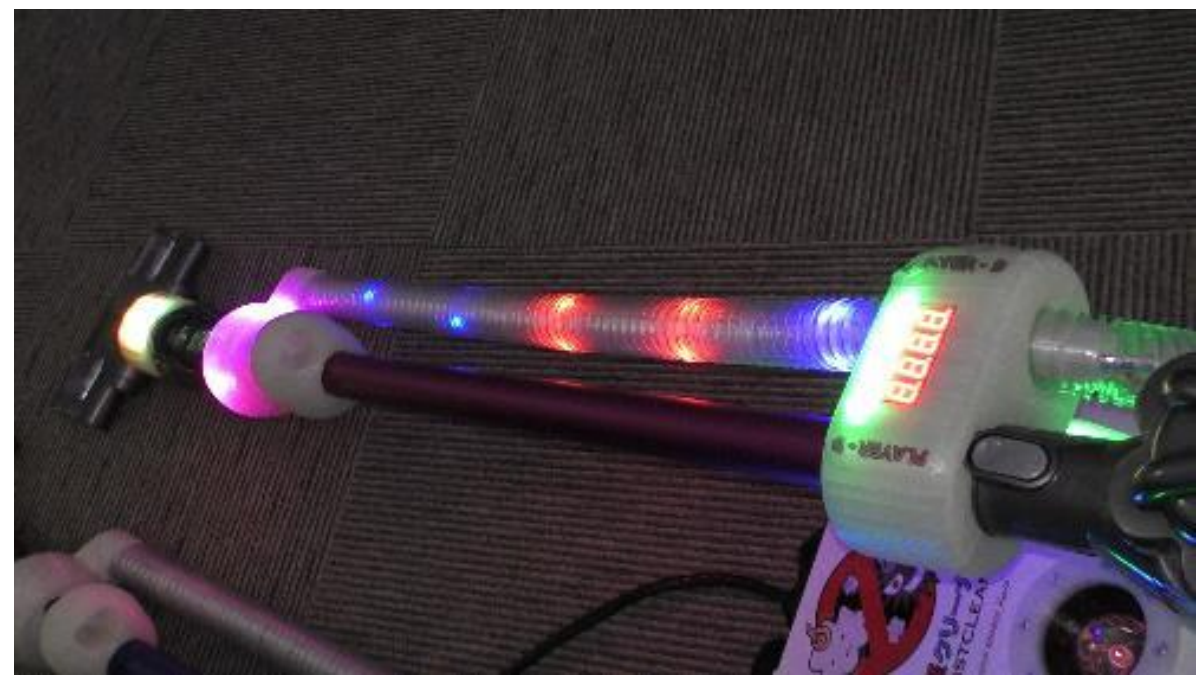

Figure 3: Display device 


\subsection{Control device}

All equipment to control the system is installed in a backpack that the user carries on his/her back. The backpack contains an Arduino Mega microcontroller board for system control, a speaker for expressing the monsters' scream, and a large fan motor to give an impression that the monster is resisting being vacuumed. In addition, the backpack contains a Bluetooth module for wireless communication with the game device (Figure 4).

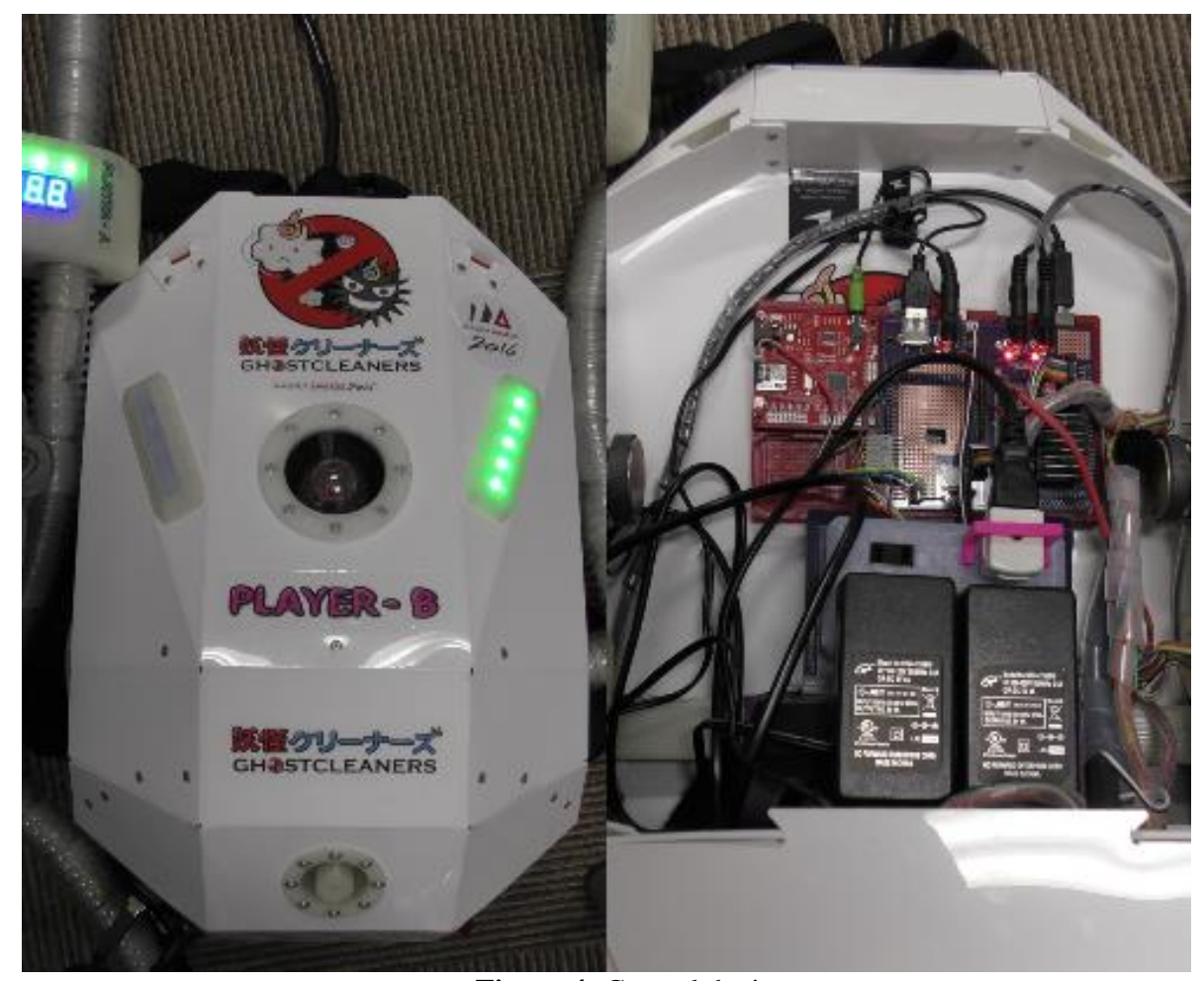

Figure 4: Control device

\subsection{Game device}

The game device is a computer that controls the status of the whole system, including information on whether devices are connected and the game level configuration. It is also used to present instructions for playing the game, the current game situation, and results. While playing the game, the players do not see the screen of the device and only use the vacuum unit. After the game ends, the game device displays the activation ratio (the areas cleaned by a player), distance moved, average speed, maximum speed, and number of monsters collected along with the total score (See Figure 5). The player with the highest score wins the game. The location and the amount of dust sucked during the game are displayed by using heat-map visualization.

In general, cleaning efficiency is improved by intensively cleaning areas that gather dust easily instead of cleaning from corner to corner every day. However, it is difficult to find out where the room is dirty. Since the heat map visualization contributes to finding out the location of dirty areas, the users can clean their room more efficiently. 


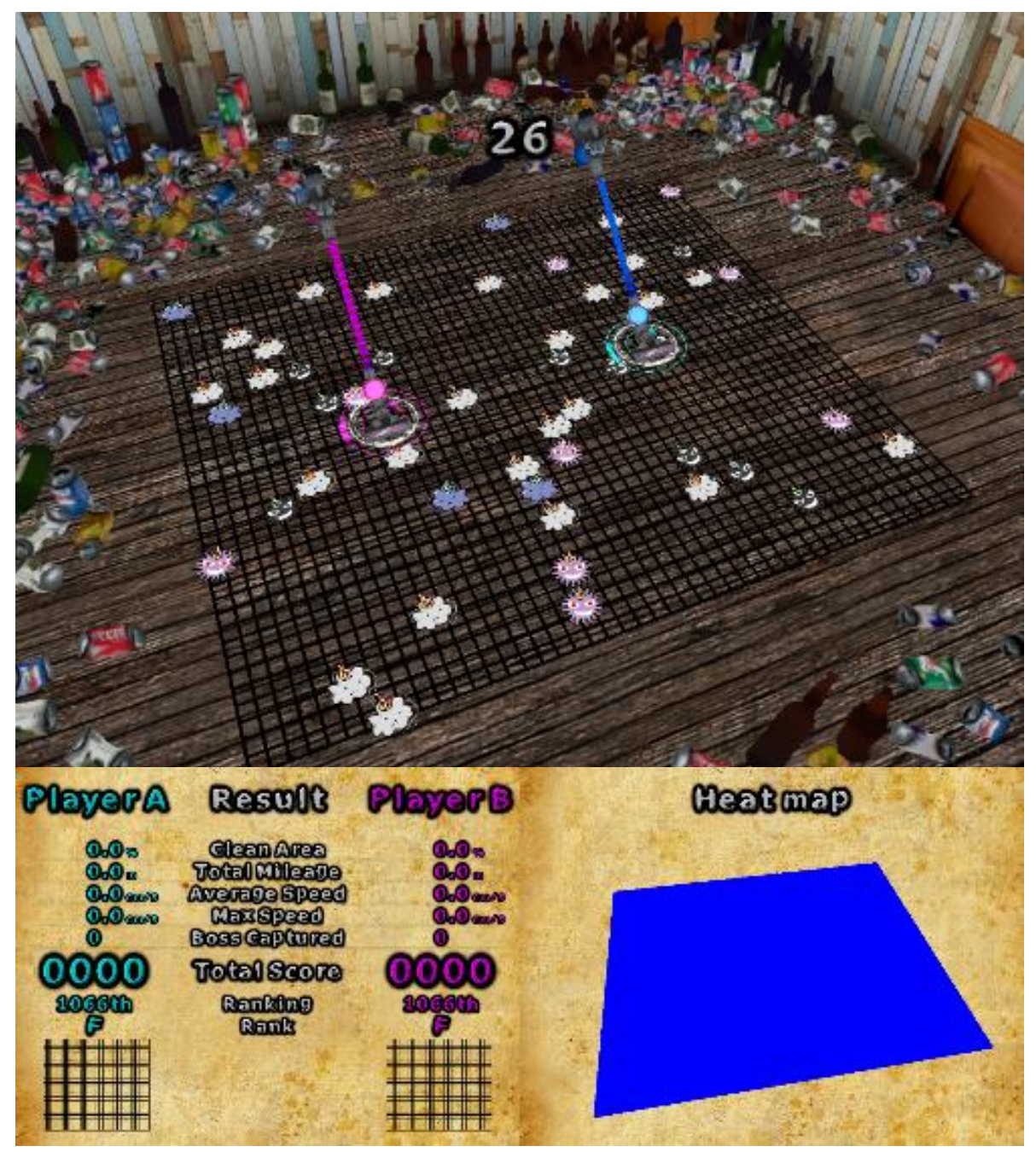

Figure 5: Game images

\section{Exhibition}

We conducted an exhibition to investigate whether children's behavior was altered by using sweeping as game content. The exhibition was conducted at the event "Kagaku Hiroba (Science Square)" held at the Kanagawa Prefecture Youth Center on June 18, 2016.

As the real dust used in the exhibition, dust collected from home could have been used. However, such dust contains a mixture of sand, mud, human hair and skin fragments, insect carcasses, ticks, mold spores, and fabrics. Therefore, it is not hygienic to scatter actual dust on the mat used in the exhibition. Thus, heat-shrink cables with a diameter of $1 \mathrm{~mm}$ were cut into $1 \mathrm{~mm}$ pieces, and those pieces were heated with a dryer to use as pseudo dust during the exhibition (see Figure 6).

In the exhibition, we observed children who actively vacuumed to collect more monsters than their opponents. Many parents said something like "I wish they could do vacuuming so diligently at home." Figure 7 shows a scene from the exhibition. 
We observed that a player moved the cleaner head rapidly soon after the start of the game. As the system detected the speed was over the limit, a warning expressing "move slowly" was presented and the LEDs were turned off, indicating that the player could not suck monsters. He noticed this soon and then moved the cleaner head slowly. This case suggests that players can learn how to use the vacuum cleaner properly through the game play. The reason why the player initially moved the cleaner's head so fast is probably that the game involved competition with another player and he wanted to win. To avoid such behavior, changing the game's rules from being competitive to being cooperative will be effective.

To guide the player to an arbitrary position, we placed a boss monster on the floor and observed the players' reactions. We observed that players moved the cleaner's head slowly to look for the boss monster.

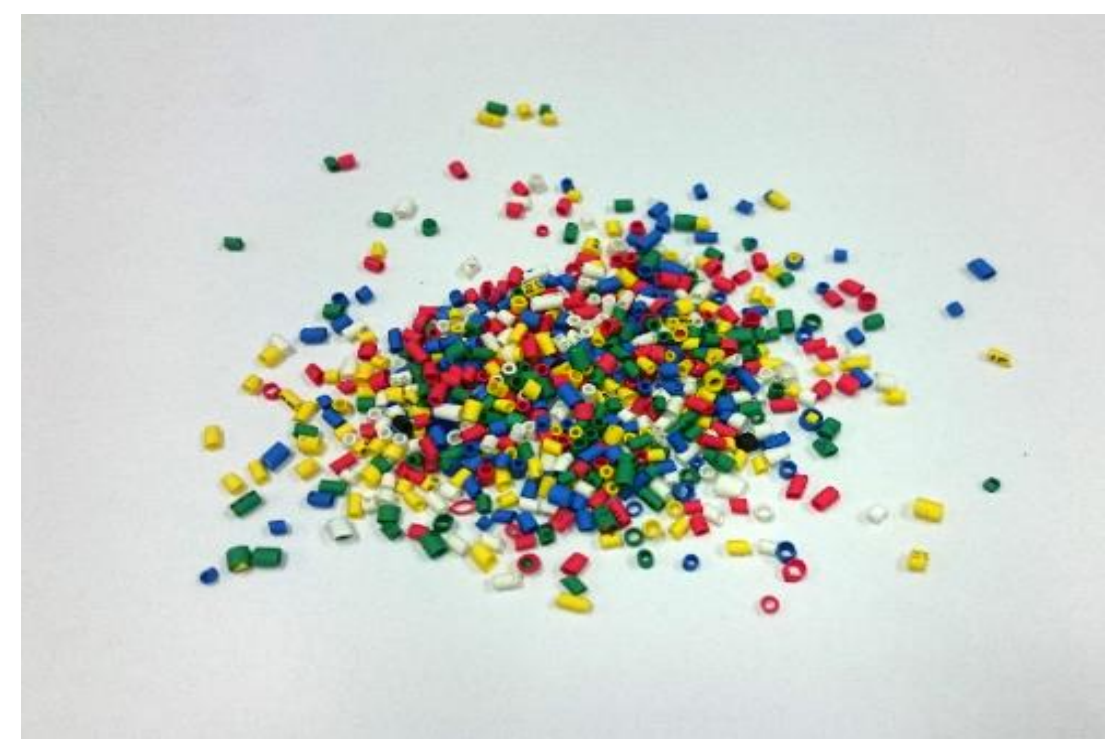

Figure 6: Small debris for exhibition

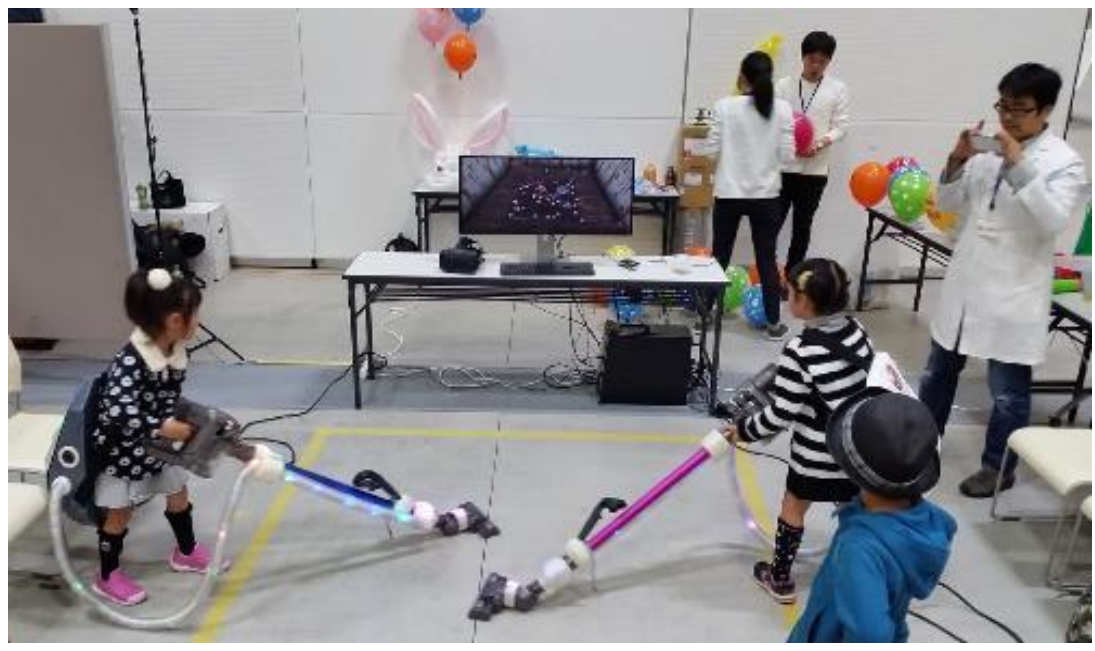

Figure 7: Demonstration of Monster Cleaner 
Parents and audience around the players also whooped, for example, "That's there!" and "I found the monster there!," indicating that the audience was involved in the game.

In the exhibition, many of the participants were primary school students. Focusing on their behavior, a difference in behavior was observed between lower-grade children and higher-grade children. Participants in lower grades were observed to hold the vacuum cleaner with one hand instead of using both hands. They seemed unfamiliar with the handling of a vacuum cleaner. They moved the vacuum cleaner on the floor rather than to suck the dust. The weight of the vacuum cleaner used was $2.3 \mathrm{~kg}$, and the total weight of the system including the sensor and display devices was $2.8 \mathrm{~kg}$. Considering the physical strength of children in lower grades of the elementary school, we noticed that this system was too heavy for them. This suggests that the system should be made lighter. Also, the trigger mechanism should be improved as some children have a weak grip power and cannot keep the trigger pressed during the game.

\section{Conclusion}

This study proposed a serious game system named Monster Cleaner that intends to motivate children to clean their room. With this system, which adds the aspect of gaming to cleaning, children can not only do cleaning while having fun but also learn to use a vacuum cleaner.

In this implementation, a dust sensor is mounted to detect dust collected in the real world. A number of vacuum cleaners equipped with dust detection sensors that notify dust suction by LED blinking are commercially available. Thus, the system can be made simpler and more versatile by acquiring the LED notification using a photocoupler.

In the future, we will focus on training players in specific uses of the vacuum cleaner. For example, we will implement a system for training players on how to clean Japanese tatami mats.

There are two types of interest: a direct interest and an indirect interest. The former is an interest in an object and/or an activity itself, and the latter is an interest that emerges from a liking toward a previously considered uninteresting object and/or action by undertaking some activities. It is suggested that the indirect interest can be evolved to a direct interest by examining the activities [9]. Our research is oriented toward an indirect-interest-based approach, and the result presented in the study suggests its usefulness. Matsumura pointed out that it is important to consider both physical aspects (e.g., system and equipment) and psychological aspects (e.g., curiosity, self-esteem) to design such efforts [10]. We intend to incorporate these enforcements and extend the research targets to other housework apart from cleaning.

\section{References}

[1]Ryoko Ogasawara, Taeko Yamaki, Koji Tsukada, Keita Watanabe, Ichiro Siio. 2007. Interactive Cleaner. In Proceedings of the Entertainment Computing 2007 (Osaka, Japan, October 1-3, 2007), 7174.

[2]Kazuki Tada, Daiki Yamaji, Kyohei Kanno, Yuya Kawabata. 2014. VACUUUUUUM. In Proceedings of the Virtual Reality society of Japan Annual Conference (Osaka, Japan, September 1820, 2013), 19.

[3]Keiko Oono, Koji Tsukada, Itiro Siio. 2013. Kadebo: A small robot attached on home appliances for joyful household chores, In Proceedings of the Entertainment Computing 2013 (Kagawa, Japan, October 4-6, 2013), 288-291. 
[4]Hirotaka Osawa, Michita Imai. 2008. Evaluation of Direct Function Explanation using Agentized Object. In Proceedings of the Interaction 2008 (Tokyo, Japan, March 3-4, 2008), 167-168.

[5]Takuya Iwamoto, Yusuke Sasayama, Mitsuo Motoki, Takayuki Kosaka. 2009. Back to the mouth. In Proceedings of the ACM SIGGRAPH 2009 Emerging Technologies (New Orleans, Louisiana, August 3-7, 2009). SIGGRAPH '09 ACM New York, NY, USA, No.4. DOI= https://doi.org/10.1145/1597956.1597960.

[6]Takayuki Kosaka, Takuya Iwamoto. 2013. Serious dietary education system for changing food preferences "food practice shooter". In Proceedings of the Virtual Reality International Conference (Laval, France, March 20-22, 2013). VRIC '13. ACM New York, NY, No. 23. DOI= https://doi.org/10.1145/2466816.2466841.

[7]Yuichi Bannai, Takayuki Kosaka, Naomi Aiba. 2014. Food Practice Shooter: A Serious Game with a Real-World Interface for Nutrition and Dietary Education. In Proceedings of the Human Interface and the Management of Information. Information and Knowledge Design and Evaluation (Heraklion, Crete, Greece, June 22-27, 2014). 8521, 139-147.

[8]Japanese Industrial Standards. 1999. Methods of measurement of performance of vacuum cleaners for household and similar use. JISC9802.

[9]Paul J. Silvia. 2006. Exploring the Psychology of Interest. 2006. Oxford University Press.

[10]Naohiro Matsumura, Renate Fruchter, Larry Leifer. 2015. Shikakeology: Designing Triggers for Behavior Change, AI \& Society, 30:419. DOI= https://doi.org/10.1007/s00146-014-0556-5 